\title{
A Magnetic Configuration Database for Wendelstein 7-X and its Application Programming Interface
}

\author{
A. Dinklage, J. Geiger, G. Kühner, M. Schmidta ${ }^{\mathrm{a}}$ Yu. A. Turkin and A. Werner \\ Max-Planck-Institut für Plasmaphysik, EURATOM Association, Greifswald, Germany \\ a present address: $A M D$, Dresden, Germany
}

\begin{abstract}
A magnetic configuration database (MCDB) storing the results and global properties from equilibrium calculations is implemented obeying physical demands. MCDB is part of W7-X databases and structured for a straight-forward upgrading or exchange of software components. Performance and reliability was tested and its applicability confirmed. The database schema allows for further extensions to different device relevant data.
\end{abstract}

Keywords: database techniques

\section{Data sources for steady-state fusion devices}

Steady-state fusion devices such as Wendelstein 7-X (W7-X) and ITER expose new challenges in information processing [1] because large amounts of data with complex interdependencies are to be handled. Data streaming during the experiment directly lead to the requirement for on-line processing and physical analyses. An example for which such processed data is to be used on-line, are electron cyclotron current drive scenarios for $\mathrm{W} 7-\mathrm{X}$ which require a transport model to be resolved for proper deposition [2]. The off-line post processing of data must face the requirements for novelty detection and validation of physical results. Non-automated approaches appear to be unsatisfactory in relation to financial costs for building large fusion devices and performing experiments.
Matching with the requirements of automation, databases are the data source technology of choice. Databases are well established and reliable tools which demonstrated availability even under changes of technologies. References in the database allow in a natural way to establish links of related data-sets. Database structures allow one to trace information processing in a natural way and the administrative effort is much less compared to distributed data sources such as file systems. The most prominent advantage of databases relevant for steady-state fusion experiments, however, results from search capabilities, which give access to availability of data or to classify results but also to trigger events, such as further analyses.

Therefore, a database based concept of information processing is the approach of choice to cope with requirements to be expected for large steady-state 
fusion devices.

Data-sets which are prerequisite for the preparation of diagnostics and for predictive modelling of Wendelstein 7-X are magnetic configurations - or more specific - the result from equilibrium calculations. In a first step, results from the MHD equilibrium code VMEC [3] are to be stored which represent basically a Fourier representation of magnetic flux surfaces. An example of which is shown in Fig. 1. As part of presently ongoing efforts of the physics

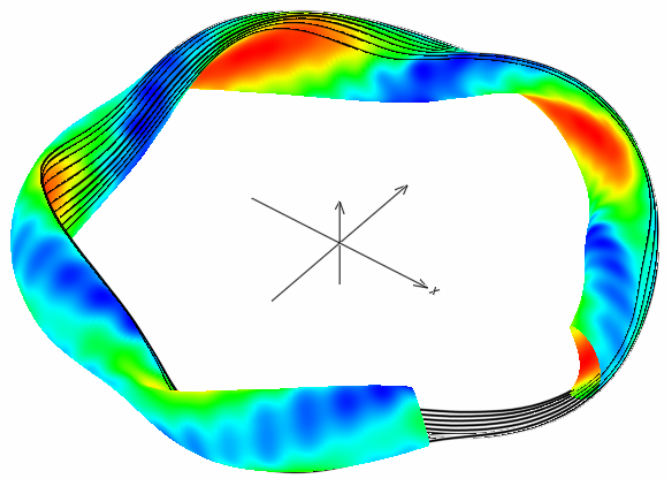

Fig.1. Reconstruction of a Wendelstein 7-X equilibrium flux surface with field lines from Fourier modes as stored in the magnetic configuration data-base. The shade of the colour indicates the variation of the modulus of the magnetic field on the flux surface shown.

preparation of Wendelstein 7-X operation, systematic calculations of the MHD equilibriums for envisaged W7-X coil parameters will be used as the basis of fast equilibrium reconstruction by means of function parameterization or neural network reconstructions [4]. Hence, the database also documents the set of calculations used for fast reconstruction approaches.

This paper describes the implementation of a database structure capable to store plasma equilibriums and to associate physically relevant parameters for qualifying the results of equilibrium calculations. In particular, the top-bottom design from physical demands is summarized and aspects resulting from the overall data processing concept of Wendelstein 7-X is highlighted. The layers of interfaces and implementation of an application programming interface (API) is outlined.

\section{Demands}

The database structure has to meet with requirements which can be ordered in their degree of details. From a technical point of view, the result of equilibrium calculations is given by a number of Fourier modes containing a cylindrical representation poloidal and toroidal modes for each flux surface. Depending on the required accuracy for subsequent calculations the number of modes may exceed a few hundred, a typical figure is $12 \times 12$ modes per fluxsurface. The flux surface is characterised by the normalized flux, the value of the rotational transform and, if applicable, characterising currents. Each equilibrium data set is made up by a bunch of flux surfaces, the number of which is one of the settings to be chosen dependent on the purpose of further processing. E.g., for detailed modelling the mesh of surfaces is finer than for scans, which are used for reconstruction databases. This adjustment is necessary due to the computational costs of equilibrium calculations. Moreover, the configuration space is hardly to be covered due to several independent input parameters (coil-current ratios) and profile functions to be given for equilibrium calculations.

A typical request to the database engine is to find the equilibrium for some set of parameters. Since the input parameter space cannot be covered densely either one has to perform a dedicated calculation or to check for a similar equilibrium calculation result. This process requires implementing an appropriate metrics on the parameter space in order to measure the vicinity to existing calculations. Moreover proper interpolation schemes are to be realised in addition. It is expected that this kind of request is of particular interest if iterations are to be done. A different motivation is due to datasets related to prominent configurations for which further modelling might be existent but is costly (e.g. transport/current drive simulations).

Further demands refer to quality parameters of calculations such as number of iterations for solving the MHD equilibrium or the convergence rate of calculations. These factors may indicate the occurrence of resonance effects making interpolations in parameter space questionable.

One key to automated approaches is traceability 
because critical parameters can be identified after analyses. Therefore, a unique description of a calculation leading to a data-set is desirable. Following an object oriented approach, a unique identification of

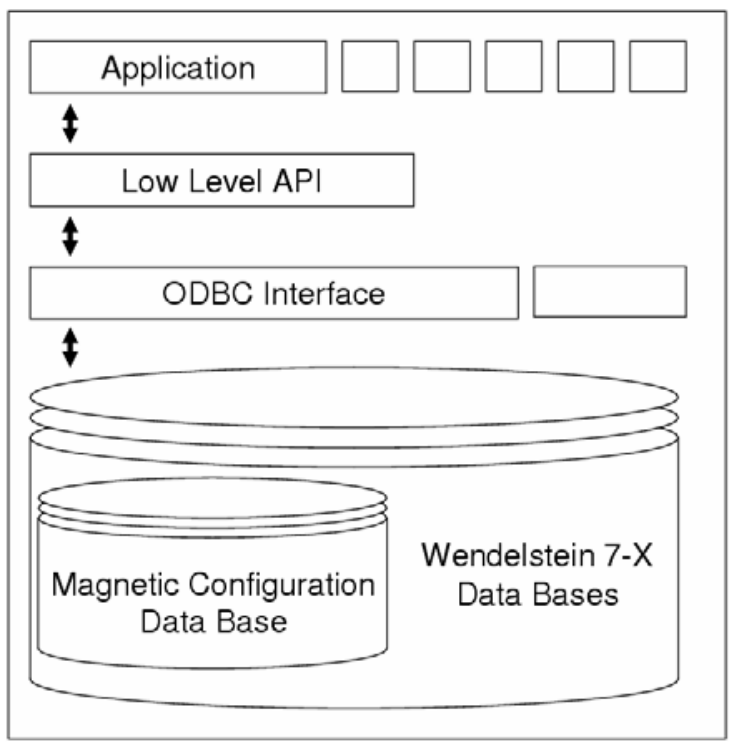

Fig.2. Schematic view on the interfaces to MCDB.

the method (here the equilibrium code) closes the set of parameters necessary for a complete description of the generation of a data set.

Since the equilibrium calculation is related to device properties, an extension to database tables containing, e.g., machine set-ups should be foreseen.

The database for magnetic configurations has to be part of the W7-X database representing the unique and only source for static data used in the experiment.

Finally, any development should ensure upgrading capabilities to highest extent with respect to the expected life-time of the devices and experience with the development of information technologies.

\section{Implementation}

\subsection{Layers of the database and its interfaces}

As shown in Fig. 2, the magnetic configuration database is part of the Wendelstein $7-\mathrm{X}$ database system. The lowest level interface allows the use of standardized drivers and an extension to different interfaces (e.g. JDBC) is kept open. The low level API is a library of $\mathrm{C}$ function for managing any of the database entries.

The basic idea of the layered structure is to allow for upgrading even of the database management system, which is an option meeting with the aforementioned requirement for possible up-grades.

\subsection{Overview on the relational database management system}

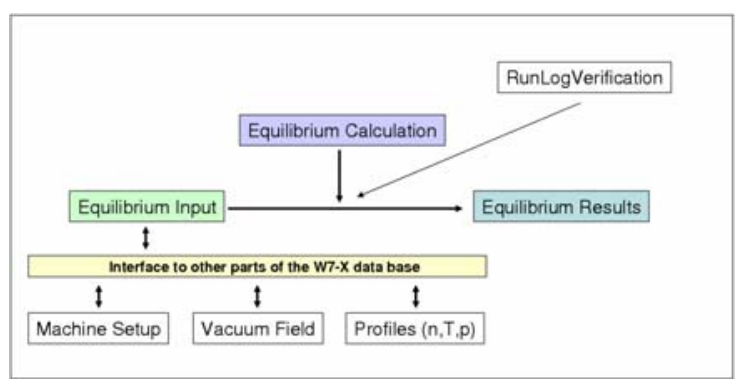

Fig. 3. Basic structure of MCDB. Arrows indicate logical relationships.

I'he structure of the database is shown in Fig.3. Each board represents a set of tables in a way outlined below. The central part of the diagram (Equilibrium Input (EI), Equilibrium Calculation (EC), Equilibrium Results (ER)) is reflecting the generic data analysis model incorporated in the W7-X raw database [5], i.e. to separate the input parameters (arguments EI) of a computation from its settings (EC), used to change the performance of a code, and the results (ER).

For a complete description the code is referenced to a unique identification in a versioning system (RunLogVerification). This provides full traceability.

Moreover, linkage to supplementary information is already implemented (via interface to other parts of W7-X database, e.g. essential parameters describing the device under consideration - this also allows usage of the database for non W7-X equilibria) or foreseen.

3.3 Hierarchy of data tables 
The database client is assumed to be primarily interested in the results of calculations. Access to any other information is provided as well. Focusing on the results, the bulk information from the equilibrium calculations is not the primary target of physically motivated search strategies rather than derived or global results. Therefore, the data tables are structured to hierarchies of logical subunits from global physical or calculation descriptive data to geometry to flux surfaces to the set of Fourier modes. The latter is implemented both in a table exposing the parameters explicitly and in a table storing the calculation results as are in binary form (BLOB). This option is superior in performance, the prior, however, allows for searches in the Fourier components. This property might be of interest if truncated versions (with respect to a threshold in the Fourier components) of one calculation result are to be compared.

\section{Technical properties}

The database implementation was performed with ORACLE 9i on an on-site database server. Drivers for the Open DataBase Connectivity (ODBC) interface were used for direct database access providing a widely and standardized solution for database access in clientserver paradigm with SQL for database requests. Drivers both for Windows (Oracle 10g) and Linux (Data Direct) were used and tested. For the application programming interface (API) a library for database table manipulation was implemented in the computer language $\mathrm{C}$ allowing a straightforward binding to object oriented $(\mathrm{C}++)$ and procedural (Fortran) codes. This choice reflects also the general philosophy to allow for upgrades in any of the layers also shown in Fig. 2 and to cope with different computer languages and operating systems.

A high level application was used for testing performance and reliability of the database system and interfaces and a low level test suite is released with the API.

The documentation consists of a set of general and introductory web sites integrated in the Wendelstein 7$\mathrm{X}$ software web documentation and the API documentation employs an automated documentation tool (dOxygen [6]). The source code is administered within a versioning system (CVS) on an on-site server.

Table 1 shows some present figures of the
Table 1 Figures of loading performance of typical equilibrium datasets (about 10 MByte/s LAN with local ORACLE 9i server on site employing the MCDB API and $25 \mathrm{MB} / \mathrm{s}$ local Hard-disk (HDD), AFS is a distributed file system for on-site file exchange at IPP) with a $3.1 \mathrm{GHz}$ PIV PC. The index $(\mathrm{t})$ refers to truncated Fourier modes, the truncation affects the storage size in the database since a variable number of Fourier modes can be stored for each flux-surface.

\begin{tabular}{|c|c|c|c|}
\hline $\begin{array}{l}\text { Sample } \\
\text { Configuration/ } \\
\text { Format }\end{array}$ & $\begin{array}{l}\text { File Size } \\
(\mathrm{MB})\end{array}$ & Place & $\begin{array}{l}\text { loading time } \\
\text { (s) }\end{array}$ \\
\hline $\begin{array}{l}\text { W7X-SC1 } \\
\text { ASCII }\end{array}$ & 19 & AFS & 115 \\
\hline $\begin{array}{l}\text { W7X-SC1 } \\
\text { ASCII }\end{array}$ & 19 & Local HDD & 10 \\
\hline $\begin{array}{l}\text { W7X-SC1 } \\
\text { binary }\end{array}$ & 10 & Local HDD & 0.6 \\
\hline $\begin{array}{l}\text { W7X-SC1 } \\
\text { BLOB }\end{array}$ & 11 & DB/LAN & 1.2 \\
\hline $\begin{array}{l}\text { W7X-SC1(t) } \\
\text { ASCII }\end{array}$ & 3.9 & Local HDD & 1.1 \\
\hline $\begin{array}{l}\text { W7X-SC1(t) } \\
\text { binary }\end{array}$ & 2 & Local HDD & 0.17 \\
\hline $\begin{array}{l}\text { W7X-SC1(t) } \\
\text { BLOB }\end{array}$ & 1.2 & DB/LAN & 0.12 \\
\hline
\end{tabular}

performance of data loading from the database giving a rate of about $10 \mathrm{MB} / \mathrm{s}$. This figure performs about 1000 times faster than loading from a distributed file system (AFS) previously used for storage of configuration files. For full configuration files, the database access is limited by LAN load. However, the database structure allows for storage of Fourier modes of interest (limited by a value threshold) which than allows loading times comparable to those from a local hard-disk for single database client access.

In addition to the figures for loading, the effectiveness for searching in the data-base has to be regarded, i.e. the administrative effort to identify parameters of equilibriums which is a resolved within a single request if data are stored in the database. 


\section{Conclusions and Outlook}

A database development for the outcome of MHD equilibrium calculations starting from expected physics requirements and technical necessities yielded a database schema which reflects the generic data analysis model for Wendelstein 7-X. Particular emphasis was led on a layered structure allowing for up-grades or even a complete exchange of components if necessary. The realisation employs standard software components allowing the usage of a wide range of compatible interfaces and applications. The development gives full traceability of equilibrium calculations in a natural way. The performance of the database and its interface was demonstrated in connection with an application depending on the processing of equilibrium data.

Based on the implementation of the database different branches of further developments appear:

First, an extension of the database system describing physical and technical properties of the Wendelstein 7-X device is planned. The priorities for such developments are oriented at the necessities for diagnostics design and optimization. Examples are a reference database for vessel components or physical databases for predictive modeling (e.g. neoclassical transport coefficients).

Second, the magnetic configuration database requires a generalization to full $3 \mathrm{~d}$ magnetic fields outside the separatrix or the results from equilibrium codes allowing for magnetic islands. In addition, a formulation for an appropriate metric for the identification of fitting equilibriums for arbitrary input parameters is to be performed.

Third, technically, the feasibility of the presented approach in case of changes in the software components has to be experienced for an ultimate conclusion.

\section{Acknowledgements}

Helpful and inspiring discussions with Henning Maaßberg are appreciated.

Programming support from 3n-mobile software (Greifswald, Germany) is gratefully acknowledged.
[1] J. B. Lister, B.P. Duval, X. Llobet, W. Spears, T.J. Fredian, M. Greenwald, J.A. Stillerman, J. How, F. Saint_laurent, J.W. Farthing, The ITER project and its data handling requirements, Proc. ICALEPS2003 (Gyeongju) TH601 (ISBN 89-954175-2-8 98420) available on-line at http://icalepcs2003.postech.ac.kr/Proceedings/PAPER S/TH601.PDF

[2] Yu.A. Turkin, H. Maaßberg, C.D. Beidler, J. Geiger, N.B. Marushchenko, Predictive transport modeling for the W7-X, Europhysics Conference Abstracts Vol. 28G (2004) P1.198, on-line at http://eps2004.clf.rl.ac.uk/pdf/P1_198.pdf.

[3] S.P. Hirshman, W.I. von Rij, P. Merkel, Three dimensional free boundary calculations using a spectral Green's function method, Comp. Phys. Comm. 43 (1986) 143.

[4] A.Sengupta, J.Geiger, P.J.McCarthy, Statistical Analysis of the equilibrium configurations of W7-X stellarator using Function Parametrization, Proc. $32^{\text {nd }}$ PES Plasma Physics Conference, Taragona, P2.047 (2005).

[5] G. Kühner, P. Heimann, S. Heinzel, Ch. Hennig, H. Kühntopf, H. Kroiss, J. Maier, J. Reetz, M. Zilker, Editor for system configuration and experiment program specification, Fus. Eng. Des. 71 (2004) 225-230.

[6] dOxygen manual: http://www.doxygen.org/index.html

\section{References}

